\title{
Enhanced patient support services improve patient persistence with multiple sclerosis treatment
}

\author{
Jane Roche' \\ Yvonne McCarry ${ }^{2}$ \\ Karen Mellors ${ }^{3}$ \\ 'Beaumont Hospital, Dublin, \\ ${ }^{2}$ Quintiles Ireland Ltd, Fairview, \\ Dublin, Ireland; ${ }^{3}$ Merck Serono \\ Ltd, Feltham, UK
}

This article was published in the following Dove Press journal:

Patient Preference and Adherence

II June 2014

Number of times this article has been viewed

ORIGINAL RESEARCH

\begin{abstract}
Background: Subcutaneous interferon beta-1a (sc IFN $\beta$-1a) therapy (44 $\mu \mathrm{g}$ or $22 \mu \mathrm{g}$, three times weekly) improves relapse rates and disability progression in patients with relapsing multiple sclerosis (MS). While early treatment with disease-modifying drugs may maximize therapeutic benefit, patients with low adherence or long treatment gaps are at increased risk of relapse. MySupport is an industry-sponsored program that provides support to patients with MS who have been prescribed sc IFN $\beta-1 \mathrm{a}$ in the UK or Republic of Ireland (ROI), via telephone and text messaging, website access, and (in some cases) face-to-face support from a dedicated MySupport Nurse. The aim of this audit was to assess if the MySupport program in the ROI could improve persistence to sc IFN $\beta$-1a therapy.
\end{abstract}

Methods: Anonymized data were supplied retrospectively from the MySupport program, for ROI patients who were registered in January 2010 to receive sc IFN $\beta$-1a three times weekly. Patients were recorded as "new" at their first drug delivery; "active", if they continued to receive scheduled deliveries; "interrupted", if their medication delivery was halted; or "stopped", if no deliveries were made for 12 months. The number of "active" patients was recorded monthly for 24 months. Results were compared with data from UK patients with MS, who were receiving National Health Service (NHS) support only, or this support plus MySupport.

Results: A greater proportion of ROI patients receiving MySupport (compared against UK patients receiving NHS support only) were on treatment at 12 months (87.8\% versus $79.3 \%)$ and at 24 months $(76.2 \%$ versus $61.8 \%)$. The odds of being on treatment were significantly greater, at all time points, for ROI patients receiving MySupport, versus UK patients receiving NHS support only $(P<0.0001)$.

Conclusion: A personalized support program, utilizing one-to-one nursing support and additional support materials, can increase the probability of patients with MS remaining on disease-modifying drug treatment.

Keywords: multiple sclerosis, interferon beta-1a, persistence, adherence

\section{Introduction}

Multiple sclerosis (MS) is a chronic, progressive, inflammatory demyelinating disease of the central nervous system, with patients typically experiencing their first symptoms between the ages of 20 and 40 years old. ${ }^{1}$ The most common clinical form is relapsing-remitting MS (RRMS), where patients usually present with a fluctuating disease course that is unpredictable and transiently remitting. ${ }^{2}$ Treatment with certain disease-modifying drugs (DMDs) can partly control MS symptoms and reduce the frequency of relapses. In addition, interferon beta (IFN- $\beta$ ) has been shown to delay the accumulation of disability over time. ${ }^{3-6}$ However, to achieve full clinical benefits, treatment should be initiated early in the disease course, ${ }^{7,8}$ and patients need to be

Correspondence: Jane Roche

Beaumont Hospital, Beaumont Road,

Dublin 9, Ireland

Tel +353 877590537

Fax $+353 \quad 17974780$

Email janeoreilly24@hotmail.com 
both "persistent" (stay on treatment for the prescribed duration) and "adherent" to therapy (act in accordance with the prescribed interval and dose). ${ }^{9}$

Poor adherence to prescribed medication is a wellrecognized problem among patients with a chronic illness requiring long-term therapy. It can lead to poor health outcomes and increased health care costs. ${ }^{10}$ Considering the chronic nature of MS, the early age of disease onset, and the requirement for lifelong therapy, it is clear that treatment adherence in patients with MS is challenging. Poor adherence to DMD therapy, and long gaps between treatment (>90 days), have been correlated with an increased risk of relapse in patients with MS. ${ }^{11,12}$ Conversely, clinical outcomes have been shown to be better when DMD therapy was continuous or near continuous. ${ }^{11,12}$ Further, an exploratory analysis of a study of subcutaneous (sc) IFN $\beta$-1a therapy in RRMS at 7-8 years' follow-up showed that the highest cumulative dose of sc IFN $\beta$-1a, and highest cumulative time of exposure to sc IFN $\beta$-1a, were associated with the best clinical outcomes, including lower annualized relapse rates and lower risk of disability progression. ${ }^{13,14}$

Good treatment adherence is dependent on a wide range of factors and interventions, including adequate patient education and a healthy relationship between the patient and health care professional, based on clear communication and good emotional/psychological support. ${ }^{15}$ In the UK, the addition of an enhanced support program for patients with MS (MySupport; Merck Serono Ltd, Feltham, UK) to standard National Health Service (NHS) care has been shown to improve patient persistence with sc IFN $\beta$-1a treatment, compared against NHS support alone (Merck Serono Ltd, data on file, 2013).

The aim of this audit was to report persistence data from patients in the Republic of Ireland (ROI) who were receiving sc IFN $\beta$-1a and had access to MySupport. A further objective was to explore, using data on file (Merck Serono Ltd, 2013), whether patients in the ROI were more likely to remain on treatment, compared against a similar population of sc IFN $\beta$-1a-treated patients in the UK who were not receiving MySupport. A case study is also presented, to illustrate how collaboration between a local MS center and MySupport in the ROI can improve patient adherence and clinical outcomes.

\section{Methods}

\section{Patient support program}

In the ROI, all patients who are treated with sc IFN $\beta$-1a collect their medication from a local pharmacy, and are registered to receive MySupport. MySupport is a post-prescription patient support program, provided by Merck Serono Ireland, to improve patients' clinical experiences and optimize treatment outcomes. In the ROI, all patients have access to a dedicated specialist field nurse (MySupport Nurse) who assists MS centers with community-based support, including home visits to assist with treatment administration. The MySupport Nurse provides face-to-face training on the injection device, with a follow-up call usually within the first day of commencing treatment. Subsequent visits are arranged to help the patient set the injection device from titration to the maintenance dose, and to provide an annual review of treatment. The MySupport Nurse can also respond directly to queries and requests for assistance from the patient or from an MS center. Until recently, all patients in the ROI could contact their MySupport Nurse directly, via their cell phone, if they experienced any problems. A telephone support line, manned by dedicated nurses, was used outside working hours, or for reordering medication. However, since March 2013, a dedicated nurse support line has been made available during the working week, and patients are encouraged to use this service. Additional support services provided by the MySupport program include a website with information on sc IFN $\beta$-1a treatment, a smartphone application, and a regular print magazine.

\section{Data collection}

In the ROI, data on patient persistence (patients remaining on treatment for the duration of the audit) were obtained anonymously from MySupport. As all patients in the ROI receive MySupport, no control group was available for determining whether patients receiving MySupport were more likely to remain on treatment than those receiving no additional support. Therefore, we used data from an audit in the UK (Merck Serono Ltd, data on file, 2013) for comparison.

In mainland UK, NHS patients receiving sc IFN $\beta$-1a can have their medication delivered directly to their home or workplace by a homecare provider. Registration with MySupport is optional. When patients register for MySupport, the homecare patient's identification number is provided by the homecare company to the support service. This means that, for each anonymized patient, there is a record of deliveries and whether they receive My Support services. Hence, data were collected on patients who had NHS support only, and on those who had NHS support plus MySupport (Merck Serono Ltd, data on file, 2013).

Data (from the ROI and UK) were collected retrospectively, only from patients who were registered in the MySupport 
program in January 2010, regardless of the date of treatment initiation or of medication possession status at the time. Patient consent was not required, as all data were anonymized and patients had been informed previously (via the original consent form) that their data might be used on an anonymous basis. At the date of first drug delivery (Month 1), patients were assigned a unique identification number and registered as a "new" patient. At each scheduled drug delivery, patients were recorded as "active", if they continued to receive scheduled deliveries; "interrupted", if their deliveries were halted; or "stopped", if no drug deliveries had been made for 12 months. Patients who stopped treatment and subsequently restarted were recorded as "new" patients and therefore they were no longer tracked. The percentage of patients remaining active was recorded every month for 24 months after the first drug delivery date.

\section{Statistical analyses}

All data were available as summarized reports (not individual patient data). At each monthly drug delivery, treatment status (active/interrupted/stopped) was recorded for all patients. The number of patients on treatment was the number of patients listed as "active". As no patients were lost to follow-up, the number of patients who were "off treatment" was calculated by subtracting the number patients listed as "active" from the total number of patients at Month 1. The odds of remaining on treatment were calculated as (patients on treatment/ total number of patients $) /(1$ - [patients on treatment/total number of patients]); 95\% Wald confidence intervals were also calculated. Odds ratios for remaining on treatment were calculated as the ratio of the odds for ROI patients receiving
MySupport, divided by the odds for UK patients receiving NHS support only. Odds ratios for remaining on treatment were also calculated using this method, for ROI patients receiving MySupport versus UK patients receiving NHS support plus MySupport. $P$-values were obtained using the chi-squared test, with the null hypothesis that the odds ratio is equal to 1 .

\section{Results \\ Patient persistence (ROI)}

A total of 604 patients were identified as receiving MySupport services in the ROI during the period 2010-2012. The numbers of patients still on treatment at $6,12,18$, and 24 months were: 577 (95.5\%), 530 (87.7\%), 499 (82.6\%), and $460(76.2 \%)$, respectively.

\section{Exploratory analysis (ROI versus control group)}

The UK audit identified 2,461 patients who were receiving NHS support only, and 2,884 patients who were receiving NHS support plus MySupport services (Merck Serono Ltd, data on file, 2013). Compared against the control group of patients receiving NHS support only, a greater proportion of ROI patients receiving MySupport were on treatment at 12 months $(87.7 \%$ versus $79.3 \%)$ and 24 months $(76.2 \%$ versus $61.8 \%$ ) (Figure 1). The odds of being on treatment were significantly greater in ROI patients receiving MySupport (compared against UK patients receiving NHS support only) at all time points after Month 1 , up to Month $24(P<0.001$, from Month 6 onwards) (Table 1). There were no significant differences in the odds of being on treatment up to Month 24

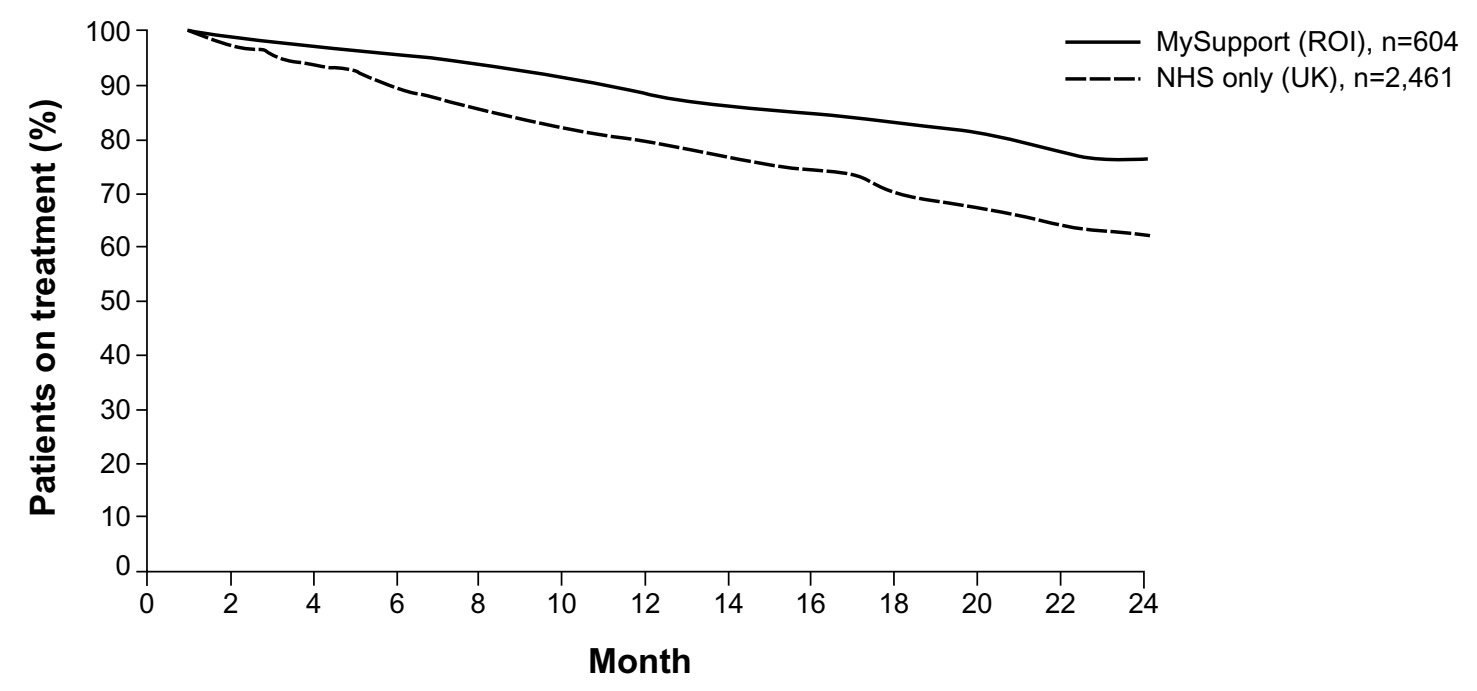

Figure I Patients remaining on subcutaneous IFN $\beta$ - I a treatment over time.

Abbreviations: IFN $\beta$-la, interferon beta-la; NHS, National Health Service; ROI, Republic of Ireland. 
Table I Odds of patients with MS remaining on subcutaneous IFN $\beta$ - I a treatment up to Month 24 when receiving MySupport versus NHS support only

\begin{tabular}{|c|c|c|c|c|}
\hline \multirow[t]{2}{*}{ Month } & \multicolumn{2}{|c|}{ Patients on treatment $(\mathrm{n})$} & \multirow{2}{*}{$\begin{array}{l}\text { Odds ratio of remaining } \\
\text { on subcutaneous IFN } \beta \text {-I a } \\
\text { treatment }(95 \% \mathrm{CI})\end{array}$} & \multirow[t]{2}{*}{$P$-value } \\
\hline & $\begin{array}{l}\text { MySupport (ROI) } \\
(n=604)\end{array}$ & $\begin{array}{l}\text { NHS only (UK) } \\
(n=2,46 I)^{a}\end{array}$ & & \\
\hline I & 604 & $2,46 I$ & - & - \\
\hline 3 & 594 & 2,357 & $2.62(1.36-5.05)$ & 0.0039 \\
\hline 6 & 577 & 2,198 & $2.56(1.70-3.84)$ & $<0.0001$ \\
\hline 12 & 530 & $\mathrm{I}, 95 \mathrm{I}$ & $1.87(1.44-2.43)$ & $<0.0001$ \\
\hline 24 & 460 & $|, 52|$ & $1.97(1.6 \mathrm{I}-2.42)$ & $<0.0001$ \\
\hline
\end{tabular}

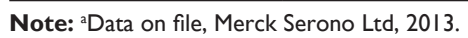

Abbreviations: $\mathrm{Cl}$, confidence interval; IFN $\beta$-la, interferon beta-la; MS, multiple sclerosis; NHS, National Health Service; ROI, Republic of Ireland.

between ROI patients receiving MySupport, and UK patients receiving NHS support plus MySupport.

\section{Case study}

The female patient is 48 years old, with a family history of RRMS. She lives with her husband, has three children at college, along with young foster children, and is employed as a part-time clerical officer. In August 2002, aged 38 years, she presented with a sudden onset of diplopia associated with nausea, vomiting, and headache, which resolved after treatment with intravenous steroids. Magnetic resonance imaging revealed multiple lesions consistent with demyelination, and oligoclonal band antibodies were detected in the cerebrospinal fluid. MS was diagnosed in February 2003, and the patient was referred to a MS clinical nurse specialist for support and education regarding her disease and treatment. The patient declined disease-modifying therapy, both initially and in April 2004, when she presented with mild right-sided weakness. However, in July 2004, after presenting with parasthesia, and after discussion with the MS clinical nurse specialist, the patient decided to begin treatment. The MS clinical nurse specialist and research nurse coordinator suggested treatment in a clinical trial setting, and the patient was educated on the commitment required for clinical trials. The patient was enrolled into the REGARD (REbif versus Glatiramer Acetate in Relapsing MS Disease) study ${ }^{16}$ in August 2004, and was assigned randomly to receive sc IFN $\beta$-1a $44 \mu \mathrm{g}$, administered three times weekly via an autoinjection device. All support at this time was given by the research nurse coordinator. During the trial period, the patient reported having missed doses, and having discarded her medication in error.

After completion of the trial, in June 2006, the patient continued with sc IFN $\beta$-1a therapy, and a MySupport Nurse visited the patient to conduct a full treatment review, providing advice on handling missing injections and rotating injection sites, and arranging follow-up, through quarterly telephone calls and a yearly visit. In subsequent telephone contacts, the patient reported being well, and taking her medication. In June 2007, the patient had an acute MS relapse and received oral steroids. The MS clinical nurse specialist and the MySupport Nurse advised the patient to remain on therapy, and provided telephone support throughout her recovery period. Contact with the MySupport Nurse increased to weekly for 4 weeks in the summer of 2008, to support the patient during retitration, as the patient had had a 1-month treatment break, due to forgetting to take her medication while on holiday.

The patient missed MS clinic visits in October 2008 and February 2009. However, during an annual visit with the MySupport Nurse in February 2009, she reported that she was adhering to her scheduled treatment. The patient had been advised by the MS clinical nurse specialist to be referred back to clinic by her general practitioner (GP). By October 2009, the patient had not attended a clinic visit for over 2 years, and was taking mefenamic acid for headache and low-dose gabapentin for neuropathic pain, as prescribed by her GP. At this time, the patient switched to using a new electronic autoinjection device (which keeps an accurate dosing log) to administer her treatment. The MySupport Nurse demonstrated the device and followed-up with the patient monthly, for 3 months. At a visit with the MySupport Nurse in June 2010, the device dosing log showed that the patient had missed 53\% of her injections. The patient indicated that she was highly motivated to adhere to treatment, but was very busy with her foster children and did not always remember to inject. The MySupport Nurse discussed methods to help the patient remember her injections, and informed the MS clinical nurse specialist of the patient's poor adherence. In October 2010, the MySupport Nurse visited the patient and found that only $66 \%$ of scheduled injections had been administered. Although the patient was concerned, she felt that her busy family life and migraine headaches were causing her to forget to take her medication. The MySupport 
Nurse and MS clinical nurse specialist liaised regarding the migraine headaches. The clinical nurse specialist then assessed the patient, and arranged an earlier appointment with the neurologist. The patient was also subsequently referred to a migraine clinical nurse specialist, and optimal changes were made to her migraine medication regimen.

At a MySupport Nurse visit in June 2011, a pattern of nonadherence was still evident, with injections frequently missed on a Friday (a busy day for the patient, owing to family commitments). The patient was advised to change her injection days to Sundays, Tuesdays, and Thursdays. During June 2011, the MySupport Nurse and MS clinical nurse specialist conducted a joint community visit with the patient, to outline a plan to increase her adherence, including setting up a reminder alarm on her cell phone for injection days and times, and reinforcing the benefits of treatment. At this time, all current MS symptoms were under control, and the device dosing log showed that the patient had taken $72 \%$ of her injections. The patient had achieved $87 \%$ compliance by June 2012 .

\section{Discussion}

These data show that a personalized, post-prescription support program using one-to-one nursing contact, plus additional support materials, can increase the likelihood of patients with MS remaining on DMD treatment. As might be expected, persistence with treatment was similar in patients in the ROI receiving MySupport compared with those in the UK receiving NHS support plus MySupport. In $\mathrm{MS}$, persistence and adherence to treatment regimens have been shown to be essential to obtaining the full benefits of prescribed DMD therapy, and to reducing the risk of relapses and increasing disability. ${ }^{11,12}$ Thus, a priority for health care teams is to ensure that all patients maximize the potential benefits of their treatment. The case study demonstrates how communication and collaboration between an MS clinical nurse specialist and MySupport Nurse helped a patient to remain on therapy, and to explore and address adherence issues. Although the reasons for nonadherence can differ, social upset and symptom exacerbation were clearly correlated with nonadherence in this case. The advantage of the device dosing log is also illustrated: the patient's actual adherence was lower than she had reported, and the availability of objective adherence data facilitated dialog between the patient and her health care professionals.

The outcomes observed in this audit are consistent with previously published support program studies in MS. In an analysis of patients with RRMS who were using glatiramer acetate, a manufacturer-provided patient support program (including injection training and copayment assistance) increased the likelihood of achieving a medication possession ratio of $\geq 80 \% .{ }^{17}$ Tan et al $^{18}$ studied the impact of an MS care management plan on clinical and economic outcomes among 4,000 patients with MS in the USA. The program included regular follow-up calls from trained nurses, provision of disease- and treatment-specific literature, and repeat prescription reminder calls. Nurses also worked as liaisons with the pharmacy, as sources of medical information, and as advocates of adherence. The program was found to be associated with improved treatment adherence and persistence, reduced risk of MS-related hospitalization, and decreased MS-related medical costs (excluding direct pharmacy costs) over 12 months.

There are a variety of reasons why patients with MS struggle to adhere to, or discontinue, their treatment, including: adverse reactions; depression; fear of the unknown/future; impact on quality of life; feeling that their treatment is not working; DMDs making patients feel worse than when they are not taking their treatment; being tired of needing regular treatment; and that the injections remind patients of their disease. ${ }^{19,20}$ In addition, patients may forget to administer their medication, which may be related to cognitive impairment resulting from the disease itself..$^{21}$ No single intervention strategy to improve adherence has been shown to be effective across all patients, conditions, and settings. ${ }^{10,22}$ Rather, success requires an approach tailored to the particular illness-related demands experienced by the patient. ${ }^{10}$ It has been recognized that the patient-health care team partnership is critical to improving adherence behaviors, and that participation, engagement, collaboration, negotiation, and compromise are key factors in encouraging patients to take responsibility for their treatment adherence. ${ }^{23}$ These partnerships foster greater patient satisfaction, improve adherence, and ultimately maximize long-term health outcomes. ${ }^{23}$ As illustrated by the case study, the range of support provided by MySupport incorporates training and advice (both face-to-face and on the telephone), and facilitates good communication between the patient and physicians, and a team-based approach among all specialist areas, with regular follow-up meetings and consistency of support for the patient. Further, the program involves close monitoring, which allows for better treatment troubleshooting and problem solving, and provides resources for patients to record their feelings, to encourage discussion with health care providers. Ultimately, the MySupport program encourages patients to take ownership of their 
own treatment adherence. In addition, use of an electronic injection device, which also stores data on the injections taken, can help patients monitor their own adherence, thus facilitating a patient-health care team dialog about persistence and adherence.

Other strategies could be used to improve treatment compliance and persistence, depending on patients' specific needs. For example, computer-tailored interventions have been shown to be effective in supporting health-related changes across a number of behaviors linked to chronic diseases. ${ }^{24}$ Berger et $\mathrm{al}^{25}$ found that the proportion of patients with MS discontinuing intramuscular IFN $\beta$-1a therapy was significantly smaller among those receiving software-guided telephone interventions $(1.2 \%)$ than among those receiving standard care $(8.7 \%)$. In addition, motivational interviewing techniques helped patients to focus preferentially on the positive aspects of intramuscular IFN $\beta$-1a therapy in making decisions about continuing treatment, and helped patients believe that therapy was an important part of their care for the upcoming 6 months. Shatil et $\mathrm{al}^{26}$ found that a homebased, computerized cognitive training program resulted in a significant improvement in three memory-based cognitive abilities among patients with MS, compared against a control group, and was also associated with increased naming speed, speed of information recall, focused attention, and visual-motor vigilance. Alternative methods could include motivational speakers and patient information days (such as those provided by the MS Society in Ireland), where specialists offer resources and information to patient groups to reinforce the benefits of treatment and discuss coping skills to relieve common MS symptoms.

Our audit had a number of limitations. As all patients with MS in the ROI receive MySupport, it was not possible to have a control group. Thus, the patient population was compared with a UK population that did not receive MySupport. A simplified version of the medication possession ratio was used (patients were recorded as "active", if they were receiving regular delivery of medication, or "interrupted", if their drug delivery was interrupted/halted). This may have overestimated adherence, as patients need to keep a backup stock of their medication. ${ }^{27}$ Moreover, it was not known how much medication patients had in stock at any time point or delivery; how regularly patients were taking their medication; or if they had discarded any medication since the previous delivery. The possibility that patients in any group may have accessed additional MS-specific support services during the course of the audit (eg, from charitable or local organizations) cannot be excluded. In addition to this, not all support systems/materials were used in the analysis and/or the case study, and no record was made of which aspects of support were used.

\section{Conclusion}

In summary, our data show that a personalized patient support program in the ROI, utilizing one-to-one nursing contact and a range of additional support materials, can increase the probability of patients with MS remaining on DMD treatment. Given that no single intervention can improve the adherence of all patients, the MySupport program allows the tailoring of assistance to each individual, fostering a supportive and productive patient-health care team relationship, and ultimately allowing patients to take responsibility for their medication regimen and adherence.

\section{Acknowledgments}

This audit was supported by Merck Serono Ltd, Feltham, UK (an affiliate of Merck KGaA, Darmstadt, Germany) and Bupa Home Healthcare, Harlow, UK. The authors thank Sharon Gladwin, Clare McNulty, and Lisa Tatler of Caudex Medical, Oxford, UK (supported by Merck Serono Ltd), for assistance with the preparation of this manuscript.

\section{Disclosure}

Jane Roche has no conflict of interest in relation to this work. Yvonne McCarry is an employee of Quintiles Ireland, which provides the MySupport Nursing Service for Merck Serono Ltd in Ireland. Karen Mellors was a contracted employee of Merck Serono Ltd, UK, at the time of manuscript preparation.

\section{References}

1. National Multiple Sclerosis Society. Who gets MS? New York: National Multiple Sclerosis Society. Available from: http://www.nationalmssociety. org/about-multiple-sclerosis/what-we-know-about-ms/who-gets-ms/ index.aspx. Accessed March 6, 2014.

2. Compston A, Coles A. Multiple sclerosis. Lancet. 2008;372(9648): 1502-1517.

3. Cohen BA, Rivera VM. PRISMS: the story of a pivotal clinical trial series in multiple sclerosis. Curr Med Res Opin. 2010;26(4):827-838.

4. Interferon beta- $1 \mathrm{~b}$ is effective in relapsing-remitting multiple sclerosis. I. Clinical results of a multicenter, randomized, double-blind, placebocontrolled trial. The IFNB Multiple Sclerosis Study Group. Neurology. 1993;43(4):655-661.

5. Jacobs LD, Cookfair DL, Rudick RA, et al. Intramuscular interferon beta-1a for disease progression in relapsing multiple sclerosis. The Multiple Sclerosis Collaborative Research Group (MSCRG). Ann Neurol. 1996;39(3):285-294.

6. Johnson KP, Brooks BR, Cohen JA, et al. Copolymer 1 reduces relapse rate and improves disability in relapsing-remitting multiple sclerosis: results of a phase III multicenter, double-blind placebo-controlled trial. The Copolymer 1 Multiple Sclerosis Study Group. Neurology. 1995;45(7):1268-1276. 
7. Comi G, Martinelli V, Rodegher M, et al. Effect of glatiramer acetate on conversion to clinically definite multiple sclerosis in patients with clinically isolated syndrome (PreCISe study): a randomised, double-blind, placebo-controlled trial. Lancet. 2009;374(9700): 1503-1511.

8. Comi G, De Stefano N, Freedman MS, et al. Comparison of two dosing frequencies of subcutaneous interferon beta-1a in patients with a first clinical demyelinating event suggestive of multiple sclerosis (REFLEX): a phase 3 randomised con trolled trial. Lancet Neurol. 2012;11(1):33-41.

9. Cramer JA, Roy A, Burrell A, et al. Medication compliance and persistence: terminology and definitions. Value Health. 2008;11(1): 44-47.

10. World Health Organization. Adherence to long-term therapies: evidence for action. Geneva, Switzerland: World Health Organization; 2003 Available from: http://www.who.int/chp/knowledge/publications/adherence_report/en/index.html. Accessed December 7, 2013.

11. Al-Sabbagh A, Bennet R, Kozma C, Dickson M, Meletiche D. Medication gaps in disease modifying therapy for multiple sclerosis are associated with an increased risk of relapse: findings from a national managed care database. J Neurol. 2008;255(Suppl 2):S79.

12. Steinberg SC, Faris RJ, Chang CF, Chan A, Tankersley MA. Impact of adherence to interferons in the treatment of multiple sclerosis: a non-experimental, retrospective, cohort study. Clin Drug Investig. 2010;30(2):89-100.

13. Kappos L, Cornelisse P, Lehr L, Kuhle J, Uitdehaag B. Relation of longterm exposure to subcutaneous interferon beta-1a with clinical outcomes in patients with relapsing-remitting multiple sclerosis: exploratory analyses from the PRISMS-15 study. Mult Scler. 2012;18(4):39.

14. Uitdehaag B, Constantinescu C, Cornelisse P, et al. Impact of exposure to interferon beta-1a on outcomes in patients with relapsing-remitting multiple sclerosis: exploratory analyses from the PRISMS long-term follow-up study. Ther Adv Neurol Disord. 2011;4(1):3-14

15. Jin J, Sklar GE, Min Sen Oh V, Chuen Li S. Factors affecting therapeutic compliance: A review from the patient's perspective. Ther Clin Risk Manag. 2008;4(1):269-286.

16. Mikol DD, Barkhof F, Chang P, et al. REGARD study group. Comparison of subcutaneous interferon beta-1a with glatiramer acetate in patients with relapsing multiple sclerosis (the REbif vs Glatiramer Acetate in Relapsing MS Disease [REGARD] study): a multicentre, randomised, parallel, open-label trial. Lancet Neurol. 2008;7(10):903-914.
17. Jones JL, Scheidt DJ, Kaushal RS, Carroll CA. Assessing the role of patient support services on adherence rates in patients using glatiramer acetate for relapsing-remitting multiple sclerosis. J Med Econ. 2013;16(2): 213-220.

18. Tan H, Yu J, Tabby D, Devries A, Singer J. Clinical and economic impact of a specialty care management program among patients with multiple sclerosis: a cohort study. Mult Scler. 2010;16(8): 956-963.

19. Patti F. Optimizing the benefit of multiple sclerosis therapy: the importance of treatment adherence. Patient Prefer Adherence. 2010;4:1-9.

20. Tremlett HL, Oger J. Interrupted therapy: stopping and switching of the beta-interferons prescribed for MS. Neurology. 2003;61(4): 551-554.

21. Devonshire V, Lapierre Y, Macdonell R, et al. The Global Adherence Project (GAP): a multicenter observational study on adherence to disease-modifying therapies in patients with relapsing-remitting multiple sclerosis. Eur J Neurol. 2010;18(1):69-77.

22. Thota AB, Sipe TA, Byard GJ, et al. Collaborative care to improve the management of depressive disorders: a community guide systematic review and meta-analysis. Am J Prev Med. 2012;42(5):525-538.

23. Martin LR, Williams SL, Haskard KB, Dimatteo MR. The challenge of patient adherence. Ther Clin Risk Manag. 2005;1(3):189-199.

24. Krebs P, Prochaska JO, Rossi JS. A meta-analysis of computer-tailored interventions for health behavior change. Prev Med. 2010;51(3-4): 214-221.

25. Berger BA, Liang H, Hudmon KS. Evaluation of software-based telephone counseling to enhance medication persistency among patients with multiple sclerosis. J Am Pharm Assoc (2003). 2005;45(4): 466-472.

26. Shatil E, Metzer A, Horvitz O, Miller A. Home-based personalized cognitive training in MS patients: a study of adherence and cognitive performance. NeuroRehabilitation. 2010;26(2):143-153.

27. Nau DP. Proportion of Days Covered (PDC) as a Preferred Method of Measuring Medication Adherence. Springfield, VA: Pharmacy Quality Alliance; 2011. Available from: http://www.pqaalliance.org/ images/uploads/files/PQA\%20PDC\%20vs\%20\%20MPR.pdf. Accessed March 11, 2013
Patient Preference and Adherence

\section{Publish your work in this journal}

Patient Preference and Adherence is an international, peer-reviewed, open access journal focusing on the growing importance of patient preference and adherence throughout the therapeutic continuum. Patient satisfaction, acceptability, quality of life, compliance, persistence and their role in developing new therapeutic modalities and compounds to

\section{Dovepress}

optimize clinical outcomes for existing disease states are major areas of interest. This journal has been accepted for indexing on PubMed Central. The manuscript management system is completely online and includes a very quick and fair peer-review system. Visit http://www.dovepress.com/ testimonials.php to read real quotes from published authors. 